\title{
Investigación en recreación, ocio y tiempo libre en Venezuela. Enfoques subyacentes
}

\author{
Research in recreation, leisure and free time in Venezuela. Underlying \\ approaches
}

*Alixon David Reyes Rodríguez, ** Eloy Altuve Mejía, \& *** Gregorio Arandia

Reyes, A., Altuve, E., \& Arandia, G. (2021). Investigación en recreación, ocio y tiempo libre en Venezuela. Enfoques subyacentes. Revista Ciencias de la Actividad Física UCM, 22(2), julio-diciembre, 1-27. http://doi.org/10.29035/rcaf.22.2.8

\section{RESUMEN}

Este trabajo presenta la primera aproximación histórico-analítica-crítica a las concepciones manejadas en la investigación sobre recreación, ocio y tiempo libre en Venezuela entre 1991 y 2010. Se empleó la metodología propuesta por el Grupo de Investigación en Ocio, Expresiones Motrices y Sociedad de la Universidad de Antioquia, Colombia, para caracterizar enfoques de investigación en el campo. Las unidades de análisis fueron: trabajos de grado, artículos de revistas, capítulos de libros, ponencias, elaborados por egresados de postgrado, docentes e investigadores de universidades venezolanas. Se consideraron: títulos de los trabajos, tipo de material, institución auspiciadora, metodología, palabras clave, autores y su procedencia. Los trabajos fueron seleccionados tras la revisión de catálogos de las instituciones y sus respectivos repositorios, consultas con directores y coordinadores de unidades y líneas de investigación, coordinadores de programas de formación avanzada, etc. Se ingresaron 162 trabajos, producidos por investigadores de varias nacionalidades en diversas universidades del país, y los resultados sugieren que la investigación en recreación, ocio y tiempo libre en Venezuela ha seguido una trayectoria marcada por enfoques de carácter positivista e instrumental en el período estudiado.

Palabras clave: ocio, recreación, tiempo libre, enfoques de investigación.

\section{ABSTRACT}

This work presents the first historical-analytical-critical approach to the conceptions handled in research on recreation, leisure and free time in Venezuela between 1991 and 2010. The methodology proposed by the Research Group on Leisure, Motive Expressions and Society of the University of Antioquia, Colombia, to characterize research approaches in the field. The units of analysis were: graduate work, magazine articles, book chapters, lectures, prepared by postgraduate graduates, teachers and researchers from Venezuelan universities. The following were considered: titles of the works, type of material, sponsoring institution, methodology, keywords, authors and their origin. The works were selected after reviewing the catalogs of the institutions and their respective repositories, after consultations with directors and coordinators of units and lines of research, coordinators of advanced training programs, etc. 162 papers were entered, produced by researchers of various nationalities at various universities in the country, and the results suggest that research on recreation, leisure and free time in Venezuela has followed a trajectory marked by positivist and instrumental approaches in the period studied.

Key words: leisure, recreation, free time, research approaches.

\footnotetext{
* Grupo de Estudios AFSYE de Pedagogía en Educación Física y Programa de Magíster en Ciencias de la Motricidad Humana de la Universidad Adventista de Chile. Chillán, Chile. https://orcid.org/0000-0001-9857-0930 | alixdavid79@gmail.com

** Centro Experimental de Estudios Latinoamericanos "Dr. Gastón Parra Luzardo" de la Universidad del Zulia. Quito, Ecuador. https://orcid.org/0000-0002-7683-0065 | eloyaltuve@hotmail.com

*** Centro Experimental de Estudios Latinoamericanos "Dr. Gastón Parra Luzardo" de la Universidad del Zulia. Maracaibo, Venezuela. gregarandia@gmail.com
} 


\section{INTRODUCCIÓN}

Uno de los temas considerados de interés en el contexto de la epistemología da cuenta de la preocupación de esta disciplina científica, esto es, el conocimiento; qué es, cómo y desde dónde se origina, cuáles son las lógicas de generación de conocimiento, cuáles son sus implicaciones en el contexto de los circuitos de producción, circulación y transferencia de conocimiento como las universidades, centros y grupos de investigación, programas de formación universitaria (pregrado y posgrado), entre otros (Arias \& Navarro, 2017, Osorio, 2007, Reyes, 2014). Se trata, en todo caso, de un tema complejo, habida cuenta la existencia de corrientes que inclinan la balanza en cuanto a enfoques, abordajes, sistemas de validación de conocimiento, etc., y es que, este mismo debate ha catalizado la emergencia de conceptos de relevancia como el de 'comunidad epistémica' (Ruiz, 2018, Villoro, 1982), 'fraternidades epistémicas' (Broncano, 2020), o 'zonas de influencia epistémica', 'geopolítica del conocer' (Giuliano, 2018), entre otros de no menor importancia.

En este orden de ideas, en el contexto de las ciencias sociales ha venido surgiendo todo un debate en torno a los posicionamientos epistémicos en la generación de investigación y de conocimiento (Castro, 2000). Esto es, la postura y el posicionamiento epistémico tienen una influencia en la investigación, en las metodologías y, por ende, en los resultados y conclusiones, además de los procesos de validación del conocimiento (Lander 2000). De acuerdo con Castro (2000), Lander (2000) y Giuliano (2018), se trata de un fenómeno que opera en el marco de las ciencias sociales de forma genérica. Siendo así, se parte en este trabajo considerando, reconociendo y reivindicando las dimensiones del ocio, la recreación y el tiempo libre, como intereses de investigación implicados en el ámbito de las ciencias sociales (Reyes, 2020), estando también transversalizados por las mismas lógicas que operan en las ciencias sociales. Además, se asume a la recreación, el ocio y el tiempo libre, como un campo de estudios en consolidación en
América Latina (Carreño et al., 2014, Osorio, 2019, Peralta et al., 2015, Quintero, 2014, 2021). Así las cosas, vale destacar que las posibilidades formativas, amén de los estudios e investigaciones que se vienen generando en los campos de la recreación, el ocio y el tiempo libre en América Latina se han venido consolidando en la región con el paso del tiempo, y más aún en lo que llevamos del siglo XXI (Reyes, 2020).

Hay países en la región en los que se destaca un acerbo importante en cuanto a la generación de conocimiento en estos campos, tales como México, Brasil, Colombia, Argentina (Tabares et al., 2014). Esto ha ido marchando al paso de la independización del campo de la recreación con respecto a otros campos (en especial, de la Educación Física y el turismo), además de las posibilidades de formación ofrecidas en cada uno de los países y a la emergencia de investigadores que vienen marcando la trayectoria de campos que están en constitución aún, esto último a pesar del amplio bagaje que puede evidenciarse en publicaciones científicas como acopio de la sistematización de la generación de conocimiento en estos contextos.

Si consideramos a Venezuela en el contexto del campo de estudios referido a la recreación, el ocio y el tiempo libre, en comparación con los países ya mencionados, tendríamos que reconocer que Venezuela ha estado aprendiendo y creciendo en esta materia de la mano de estas naciones, con el apoyo y acompañamiento constante de sus investigadores y experiencias, además de lo que ha significado el desarrollo del campo en términos de posibilidades como lo atestiguan factores tan importantes como el Plan Nacional de Recreación para el Vivir Bien, la Ley Orgánica de Recreación, el Movimiento Nacional de Recreadores, desarrollo de programas de formación avanzada en recreación, centros y líneas de investigación, entre otros (Reyes, 2015).

Históricamente, en Venezuela, la universidad ha sido el organismo donde, desde sus centros y núcleos de investigación, de manera casi exclusiva, se ha realizado la investigación existente sobre 
recreación, incorporando en fecha reciente el ocio y el tiempo libre como objetos de estudio (Reyes, 2020). Por lo tanto, dado que la generación de conocimiento se entiende como un acto de construcción social (Mangez \& Mangez, 2011), y que, la publicación implica también un acto de producción social (Jandrić \& Hayes, 2019), el estudiar el producto investigativo universitario significa, prácticamente, abordar el análisis de la investigación sobre el tema en todo el país, dadas las condiciones y las formas cómo se produce conocimiento en estos campos en Venezuela. Para Osorio (2014):

La problematización de la recreación y el ocio hoy, nos plantea exigencias epistémicas y metodológicas que nos permitan ampliar el horizonte de análisis de dichos fenómenos, no tan solo en virtud de los profundos y acelerados cambios sociales, culturales, económicos, sino también de cómo se han construido sus significados y se ha producido conocimiento históricamente (p. 30).

Precisamente, con base en estos planteamientos, se hace preciso situar la investigación y la producción de conocimientos en el campo de estudio de la recreación, el ocio y el tiempo libre, a fin de considerar cuáles han sido las lógicas que han guiado históricamente estos procesos.

Existen ejercicios de investigación que abordan, de alguna manera, intereses de investigación asociados a la conceptualización de la recreación y otras categorías como ocio, lazer, tiempo libre. Por ejemplo, en un estudio realizado por Quintero (2021), se fueron considerando las tendencias de abordaje de la recreación en América Latina, entendiéndose de partida la recreación como un campo de estudios, conclusión que respaldada por la consulta realizada por el autor a referentes latinoamericanos de la recreación. En un trabajo realizado por Carreño et al. (2019), en relación con tendencias de investigación en la formación docente en Educación Física y Recreación, los autores advierten que la recreación se concibe y se manifiesta como un campo de investigación particular. Esto viene muy de la mano de planteamientos realizados en torno a la teoría de los campos en Bourdieu (1999). Está también el caso del trabajo realizado por Conde et al. (2016), quienes hacen un análisis conceptual de la recreación, el ocio y el tiempo libre en relación con el empleo de estos como posibilidades educativas no formales en Uruguay. También se ha ubicado un trabajo publicado por Benítez et al. (2015) en relación con la evolución del concepto de recreación y sus beneficios en poblaciones diferentes. Gomes (2014), abordó una investigación en relación con referentes conceptuales en los campos del ocio y la recreación en América Latina, determinando posicionamientos desde el pensamiento de la modernidad y la posmodernidad. Elizalde \& Gomes (2010), con un trabajo sobre los conceptos y abordajes de las categorías 'ocio' y 'recreación' en América Latina, proponen, desde un análisis crítico, una resignificación de los fenómenos. Guerrero (2009), genera una investigación a partir de la cual se describe el papel de la investigación en la formación en programas de pregrado y posgrado en Venezuela. Duque et al. (2008), hicieron una investigación sobre los fundamentos conceptuales del ocio crítico desde una perspectiva latinoamericana y, Waichman (1998), ofrece también un análisis de los enfoques teóricos y prácticos de la recreación en América Latina. Ahora, si bien es cierto que existen investigaciones en el campo, no es menos cierto que, así como los últimos trabajos citados, no son muchos los trabajos que se desarrollen desde una perspectiva crítica en relación con los orígenes y las tendencias en la producción de conocimiento en el campo de la recreación, el ocio, el tiempo libre y lazer en América Latina. Tal y como se demostrará posteriormente, los resultados de esta investigación avalan que, ciertamente, ha comenzado a generarse investigación desde otras perspectivas, aunque históricamente ha prelado una investigación positivista y de carácter instrumental. De hecho, tal y como sostiene Berkin (2019), en ciencias sociales, la investigación venía siendo generada desde un paradigma que prioriza 
El control de los métodos y los resultados de la ciencia con criterios científicos hegemónicos, genera resultados que no sólo no actualizan el conocimiento, sino que no responden a los nuevos y viejos problemas de la producción horizontal del conocimiento que nos aquejan (pp. 23-24).

Esa situación, de la que se adjudica a las ciencias sociales se ha estado replicando en la investigación que se ha hecho en América Latina con respecto al campo de la recreación, el ocio y el tiempo libre. En Venezuela no ha sido diferente (Reyes, 2020). Dadas estas condiciones, el objetivo de este trabajo pasa por sistematizar la producción de conocimiento en recreación, ocio y tiempo libre, a partir de una aproximación histórico-analíticacrítica a las concepciones manejadas en la investigación sobre este campo en Venezuela. Y para ello, se ha de considerar la propuesta de enfoques de investigación que ya planteara Habermas (1988), esto es, el enfoque empíricoanalítico, el enfoque histórico-hermenéutico y el enfoque crítico-social.

De acuerdo con Habermas (1988), el enfoque empírico-analítico asoma un interés técnico que logra consolidar a partir de la premisa de la objetividad y la consiguiente verificación de las proposiciones, considerando la predicción, la manipulación y control de las variables como elementos fundamentales (Cifuentes, 2011, Vasco, 1985). La propuesta de encuadramiento de Habermas de este enfoque es el equivalente a una metodología cuantitativa, subsidiaria de la lógica del positivismo y el neopositivismo (Coello et al., 2012, Ramos, 2015), o como la destaca Ortiz (2015), una metodología hipotético-deductiva. Luego vendría el enfoque histórico-hermenéutico, que, en Habermas, apunta a un interés práctico desde la comprensión. Asoma la comprensión, la interpretación y la adjudicación de sentidos (Vasco, 1985). Equivale a una metodología cualitativa (Coello et al., 2012, Ramos, 2015), o también reconocida como una metodología interpretativa-comprensiva. $\mathrm{Y}$, finalmente se encuentra el enfoque crítico-social, que en Habermas tiene un interés emancipatorio, y que apunta a la intervención de la realidad para su transformación (Vasco, 1985). Equivale a la metodología mixta o cuali-cuantitativa (Coello et al., 2012, Ramos, 2015), o conocida también como metodología transformacional (Ortiz, 2015).

\section{METODOLOGÍA}

Este trabajo se abordó a partir de una metodología documental-descriptiva de carácter retrospectiva, propuesta por el Grupo de Investigación en Ocio, Expresiones Motrices y Sociedad de la Universidad de Antioquia, Colombia, en la que se caracterizan los enfoques de investigación en los campos de conocimiento ya contextualizados, y que han sido publicados en trabajos de Tabares (2011) y Tabares et al. (2014). Esta metodología implica, a su vez, una clasificación de enfoques ya generada por la propuesta de Habermas (1988), partiendo de una revisión documental (Codina, 2020).

Se ha trabajado con un corpus documental referente a la producción de conocimiento en el campo de estudios de la recreación, ocio, lazer y tiempo libre, generada en Venezuela. Los criterios de inclusión fueron: investigaciones tipificadas como trabajos de grado (especialización, maestría y doctorado), trabajos de ascenso, artículos publicados en revistas científicas, conferencias o ponencias publicadas en memorias de eventos, libros, capítulos de libros; que hayan sido realizados entre 1991 y 2010; documentos apadrinados por instituciones y/o editoriales nacionales o extranjeras, siempre y cuando se tratase de trabajos relacionados con el contexto de estudio en Venezuela. En este sentido, en una primera búsqueda, fueron seleccionados los trabajos tras revisar los catálogos de las universidades e instituciones respectivas, aunando a ello los respectivos repositorios universitarios, y tras consultas con directores y coordinadores de unidades y líneas de investigación, coordinadores de programas de formación avanzada en recreación, en educación y en Educación Física. Así, los trabajos considerados para el presente análisis fueron: 
- Todas las tesis de la Maestría en Recreación de la Universidad del Zulia (LUZ);

- Tesis de la Maestría en Enseñanza de la Educación Física de la UPEL, aquellas tesis cuyos títulos tienen al menos uno de estos términos: ocio, tiempo libre y recreación (o palabras asociadas como recreo, recreativo, recreacional, etc.); el mismo criterio se aplicó para incluir trabajos de las demás universidadesy, en general, en todo el país.

- Trabajos publicados en escenarios internacionales, incluyendo aquellos que, independientemente de su título, abordan el objeto de estudio.

Los productos analizados son, principalmente, trabajos de grado (especialización, maestría, doctorado), artículos de revistas, capítulos de libros, ponencias, etc., todos elaborados por egresados de postgrados y por docentes e investigadores de diversas universidades venezolanas.

\section{RESULTADOS}

De acuerdo con Velasco (2018), "el proceso de creación del conocimiento científico presenta tres partes fundamentales, a saber: la inferencia que se realiza sobre la base de los datos manejados, la interpretación de la información así obtenida, y los resultados que son generados" (p. 47). En tal sentido, este trabajo presenta esta estructura lógica y metodológica para la presentación de resultados y su análisis.

Al iniciar entregando resultados, vale destacar que, en 1991 se producen las primeras tesis de la Maestría en Recreación de la Universidad del Zulia (LUZ), siendo esta la primera experiencia de postgrado en el área en Venezuela, y las tesis de la Maestría en Enseñanza de la Educación Física de la Universidad Pedagógica Libertador-Instituto Pedagógico de Caracas (UPEL-IPC) que abordan el objeto de estudio.

Los resultados de la investigación tras la revisión documental quedan plasmados en la siguiente tabla.
Tabla 1

Tipo de materiales.

\begin{tabular}{|ccc}
\hline TIPO DE MATERIAL & $F$ & $\%$ \\
\hline Artículo & 7 & 4,32 \\
\hline Libro & 4 & 2,47 \\
\hline Memoria & 3 & 1,85 \\
\hline Tesis (TGM, TD, TA) & 135 & 83,33 \\
\hline Otros & 13 & 8,03 \\
\hline Total & 162 & $100 \%$ \\
\hline
\end{tabular}

Fuente: Elaboración propia.

La mayor producción está orientada hacia los trabajos de grado (especialización, maestría y doctorado), con proporciones reducidas entre tipos de textos como artículos en revistas, libros, memorias de eventos y otros tipos de materiales. La categoría 'Otros' se agrega, habida cuenta que se ha tratado de posters (presentados en congresos nacionales e internacionales) entregados en forma de catálogo, y que son producto de investigación. 
Tabla 2

Instituciones de adscripción.

INSTITUCIONES

ADSCRIPCIONES

Universidad del Zulia

50

30,49

\begin{tabular}{lcc}
\hline UPEL-Instituto Pedagógico de Caracas & 55 & 33,54 \\
UPEL-Instituto Pedagógico de Maturín Antonio Lira Alcalá & 18 & 10,98 \\
\hline Fe y Alegría & 1 & 0,61 \\
\hline UPEL-Instituto Pedagógico de Barquisimeto Luis Beltrán Prieto Figueroa & 1,83 & 8,54 \\
\hline UPEL-Instituto Pedagógico de Maracay & 1 & 0,61 \\
\hline UPEL-Instituto Pedagógico Rural Gervasio Rubio & 3 & 1,83 \\
\hline Universidad Simón Bolívar & 1 & 0,61 \\
\hline Universidad de la Plata & 1 & 0,61 \\
\hline Red Socialista & 1 & 0,61 \\
\hline Universidad Nacional Experimental Francisco de Miranda & 1 & 0,61 \\
\hline APDASC & 1 & 0,61 \\
\hline UPEL-Instituto Pedagógico Rural El Mácaro & 2 & 1,22 \\
\hline Universidade Federal Minas Gerais & 1 & 0,61 \\
\hline Asociación Científico Cultural en Actividad Física y Deporte & 164 & 0,61 \\
\hline Editorial Apicuri & 1 & 0,61 \\
\hline Universidad Deportiva del Sur & 1 & 0,61 \\
\hline Confederación Deportiva Autónoma de Honduras & 1 & 4,88 \\
\hline Sin Adscripción & $100 \%$ \\
\hline Total & 1 & 1 \\
\hline
\end{tabular}

Fuente: Elaboración propia.

Las instituciones que cuentan con mayor producción en este contexto son: la UPEL-IPC, LUZ, y cierra el Instituto Pedagógico de Maturín Antonio Lira Alcalá (UPEL-IPMALA). Vale destacar que hay un trabajo que tiene tres autores (por lo que se duplica un par de filiaciones).
Tabla 3

Procedencia de autores(as).

\begin{tabular}{lc|c}
\hline NACIONALIDAD & AUTORES(AS) & $\%$ \\
\hline Guatemala & 1 & 0,61 \\
\hline Venezuela & 153 & 93,29 \\
\hline Colombia & 8 & 4,88 \\
\hline Honduras & 1 & 0,61 \\
\hline Chile & 1 & 0,61 \\
\hline Total & 164 & $100 \%$ \\
\hline
\end{tabular}

Fuente: Elaboración propia. 
Destaca la nacionalidad venezolana en su mayoría (93,29\%), al tiempo que vale la pena mencionar la afluencia de investigadores de otros países como Colombia, Guatemala, Chile y Honduras.

Tabla 4

Sexo de los(as) autores(as).

\begin{tabular}{ccc}
\hline SEXO & $F$ & $\%$ \\
\hline Hombres & 85 & $51,83 \%$ \\
\hline Mujeres & 79 & $48,17 \%$ \\
\hline Total & 164 & $100 \%$ \\
\hline
\end{tabular}

Fuente: Elaboración propia.

Tabla 5

Enfoques de investigación y períodos.

\begin{tabular}{lccc} 
ENFOQUES & $F$ & $\%$ & PERÍODOS \\
Empírico-analítico & 77 & 47,53 & 1er Período: 1991-1999 \\
Histórico-hermenéutico & 54 & 33,29 & 2 2 Período: 2000-2007 \\
Crítico-social & 31 & 19,14 & 3er Período: 2008-2010 \\
Total & 162 & $100 \%$ & -- \\
\hline
\end{tabular}

Fuente: Elaboración propia.

En cuanto a los enfoques de investigación, tenemos que el empírico-analítico ha sido el enfoque de mayor presencia, seguido por el histórico-hermenéutico y cerrando con el enfoque crítico-social, lo que ha permitido periodizar el reflejo en la predominancia. Además de las tablas presentadas anteriormente, hay otros resultados que comentar tras un análisis mucho más detallado.
En la Tabla 4, puede advertirse que no hay una diferencia pronunciada entre hombres y mujeres en cuanto a la producción de conocimiento a partir de investigación en el campo de estudios asociado al ocio, la recreación y el tiempo libre en Venezuela. 


\section{Primer período: 1991-1999}

En el período comprendido entre los años 1991 y

1999, se presentan 77 trabajos (47,53\% del total).

\section{Tipo de material e institución que auspicia la investigación:}

Tabla 6

Caracterización de la producción de conocimiento en el período 1997-1999.

\begin{tabular}{llcc}
\hline Tipo de material & Institución & FR & $\%$ \\
\hline Trabajo de grado (Maestría en recreación) & LUZ & 48 & 29,62 \\
\hline Trabajo de Grado (Maestría en Educación Física) & UPEL-IPC & 21 & 12,96 \\
$\begin{array}{l}\text { Trabajo de Grado (Maestría en Enseñanza de la Educación } \\
\text { Física) }\end{array}$ & UPEL-IPMALA & 2 & 1,24 \\
$\begin{array}{l}\text { Trabajo de Grado (Maestría en Enseñanza de la Educación } \\
\text { Física) }\end{array}$ & UPEL-IPB & 1,24 \\
$\begin{array}{l}\text { Ponencia [I Congreso Nacional de Investigación del Turismo y } \\
\text { la Hospitalidad (Venezuela)] }\end{array}$ & USB & 1 & 0,61 \\
\hline Artículo (Revista Colección Procesos Educativos) & Fey Alegría & 1 & 0,61 \\
\hline Libro & Editorial Fuser & 1 & 0,61 \\
\hline Artículo (Revista Digital Educación Física y Deportes) & Sin filiación & 1 & 0,61 \\
\hline
\end{tabular}

Fuente: Elaboración propia.

Como puede advertirse, 73 de los documentos (45,06\%) son trabajos de grado de universidades, uno $(0,61 \%)$ es un libro para una editorial independiente, y dos (1,24\%) son artículos de revista. Además, entre el 100\%, se tiene que, 74 trabajos son auspiciados por las universidades (45,68\%), uno $(0,61 \%)$ es auspiciado por una editorial independiente, uno $(0,61 \%)$ por una revista digital, y uno $(0,61 \%)$ por Fe y Alegría.

\section{Término resaltante y/o destacado en el título}

Tenemos que: a) En 47 (29,01\%) se destaca el término 'programa' o 'proyecto', o ‘planes'; b) En 11 $(6,80 \%)$ se resalta el término 'acciones-actividades'; c) Según el título, 10 (6,17\%) son mediciones de relación entre variables; d) dos trabajos (1,24\%) resaltan el término 'metodología de medición'; e) cuatro (2,47\%) destacan la palabra 'formación'; f) tres (1,85\%) vienen desarrollados con criterios analíticos un poco más amplios.

Observaciones:

- Los títulos de 74 (45,68\%) resumen o reducen la investigación a lo operativo, a la ejecución de acciones: programas, proyectos, actividades, diseño, elaboración de planes, medición. Este contenido no aparece explícito en los títulos de los tres (1,85\%) restantes.

- De los trabajos cuyo título no alude explícitamente la ejecución de acciones, uno $(0,61 \%)$ es auspiciado por Fe y Alegría, uno fue publicado por la Revista Digital Educación Física y Deportes $(0,61 \%)$ y uno por la UPEL$\operatorname{IPC}(0,61 \%)$. 


\section{Presencia de los términos ocio, recreación y tiempo libre en los títulos}

\subsection{Ocio:}

Ninguno de los trabajos lo incluye en su título.

\subsection{Recreación}

Palabras derivadas como recreo, recreacional, etc., aparece como único término en los títulos de 43 trabajos $(26,54 \%)$.

El término 'recreación' aparece junto a otro(s) término(s) en los títulos de 23 (14,20\%) trabajos, asociado con: a) Tiempo libre, en dos (1,24\%); b) Deporte participativo, en uno (0,61\%); c) Deporte para todos, en uno (0,61\%); d) Educación Física y deporte para todos, en uno (0,61\%); e) Educación Física, y deporte, en seis (3,70\%); f) Educación Física, en dos (1,24\%); g) Turismo, en uno (0,61\%); h) Actividades físicas, en cuatro (2,47\%); I) Deporte, en tres (1,85\%); j) Ambientales, en uno (0,61\%); k) Deporte y salud, en uno $(0,61 \%)$

\subsection{Tiempo libre:}

a) Aparece como único término en los títulos de siete trabajos (4,32\%); b) Únicamente aparece asociado con el término recreación: títulos de dos $(7,24 \%)$.

3.4. En nueve trabajos (5,56\%) no aparece ninguno de los términos considerados (ocio, recreación, y tiempo libre) en la investigación. El término destacado en el título es la siguiente: a) Deporte participativo, en dos (1,24\%); b) Deporte para todos, en cuatro (2,47\%); c) Educación Física, en uno (0,61\%);

d) Deporte, en uno (0,61\%); e) Otro, en uno (0,61\%).

\section{Observaciones:}

- Como complemento de expresiones 'uso' o 'buen uso' está presente de manera muy limitada el tiempo libre en los títulos. Si se agrega la ausencia total de la palabra ocio, esto significa que el centro de las investigaciones es la recreación, presente exclusivamente en los títulos de 43 trabajos (26,54\%): asociada a otro término en otros 23 $(14,20 \%)$ y ausente de $10(6,17 \%)$

\section{Autores(as)}

- 65 autores(as) son venezolanos(as), y 12 son extranjeros(as), de los cuales: nueve son de nacionalidad colombiana, uno es guatemalteco, hay un hondureño y un chileno. De los extranjeros, dos residían y ejercían como profesores en Venezuela desde antes de cursar la Maestría en Recreación en LUZ y continuaron residiendo y ejerciendo la docencia universitaria después de egresar.

- Resulta llamativo la baja cantidad de autores(as) activos(as) en investigación. 


\section{Segundo período 2000-2007}

En el período comprendido entre el año 2000 y el año 2007, se presentan 54 trabajos (33,33\% del total), de los cuales:

\section{Tipo de material e institución que auspicia la investigación:}

Tabla 7

Caracterización de la producción de conocimiento en el período 2007-2007.

\begin{tabular}{|c|c|c|c|}
\hline Tipo de material & Institución & FR & $\%$ \\
\hline Trabajo de Grado (Maestría en Educación Física) & UPEL-IPC & 21 & 12,96 \\
\hline $\begin{array}{l}\text { Trabajo de Grado (Maestría en Enseñanza de la Educación } \\
\text { Física) }\end{array}$ & UPEL-IPMALA & 15 & 9,26 \\
\hline $\begin{array}{l}\text { Trabajo de Grado (Maestría en Enseñanza de la Educación } \\
\text { Física) }\end{array}$ & UPEL-Maracay & 9 & 5,56 \\
\hline $\begin{array}{l}\text { Ponencia (IV Congreso de Investigación Turística en el Sureste } \\
\text { Asiático-Tailandia, y III Congreso Latinoamericano d } \\
\text { Investigación Turística-Chile) }\end{array}$ & USB & 2 & 1,24 \\
\hline $\begin{array}{l}\text { Trabajo de Grado (Maestría en Enseñanza de la Educación } \\
\text { Física) }\end{array}$ & UPEL-IPB & 1 & 0,61 \\
\hline Trabajo de Grado (Maestría en Educación Física) & UPEL-IPRGR & 1 & 0,61 \\
\hline $\begin{array}{l}\text { Trabajo de Grado (Maestría en Enseñanza de la Educación } \\
\text { Física) }\end{array}$ & UPEL-IPRM & 1 & 0,61 \\
\hline Trabajo de Grado (Maestría en Recreación) & LUZ & 1 & 0,61 \\
\hline Artículo (Revista Digital Educación Física y Deportes) & Sin filiación & 1 & 0,61 \\
\hline $\begin{array}{l}\text { Conferencia (Congreso Olímpico de Ecuador Deporte para } \\
\text { todos. Asociación de Municipalidades del Ecuador, capítulo } \\
\text { Guayas y Comité Olímpico) }\end{array}$ & Sin filiación & 1 & 0,61 \\
\hline Ponencia (I Simposio Internacional de Recreación) & UPEL-IPB & 1 & 0,61 \\
\hline TOTALES & & 77 & 33,29 \\
\hline
\end{tabular}

Fuente: Elaboración propia.

Observaciones:

- 49 trabajos $(30,25 \%)$ son trabajos de grado de universidades; tres (1,85\%) son ponencias presentadas en congresos internacionales y nacionales, uno $(0,61)$ es un artículo presentado en una revista digital, y uno $(0,61 \%)$ es un simposio presentado en una universidad nacional.

\section{Término resaltante y/o destacado en el título}

Tenemos que: a) En 15 (9,26\%) se destaca los términos: 'programa', 'proyecto', o 'planes'; b) En 23 (14,20\%) se resalta el término: 'acciones-actividades'; c) ocho $(4,94 \%)$ son mediciones de relación entre variables; d) dos (1,24\%) incluyen en el título 'Concepto de ocio, tiempo libre y recreación' y son del mismo autor; e) dos (1,24\%) aluden en el título: 'diseño de áreas de recreación'; f) en un trabajo $(0,61 \%)$ se resalta en su título 'propuesta de modelo 
de libro... administración de la EF, D y R'; g) uno $(0,61 \%)$ de los trabajos se refiere a una ‘propuesta para la reforma constitucional'; h) uno $(0,61 \%)$ de los títulos alude a la formación de talento humano en recreación; i) uno (0,61\%) está trabajado con criterios analíticos un poco más amplios.

Observaciones:

- Los títulos de 50 trabajos (30,86\%) resumen o reducen la investigación a lo operativo, a la ejecución de acciones: programas, proyectos, actividades, diseño, elaboración de planes, medición, propuestas. Este contenido no aparece explícito en los títulos de los cuatro $(2,50 \%)$ restantes.

- De los trabajos cuyo título no alude explícitamente la ejecución de acciones, dos son auspiciados por la USB (1,24\%), de los que, uno $(0,61 \%)$ es una conferencia presentada en el Congreso Olímpico de Ecuador, y el otro (0,61\%) es un simposio auspiciado por la UPEL.

\section{Presencia de los términos ocio, recreación y tiempo libre en los títulos}

3.1. Ocio: En ninguno de los trabajos aparece en solitario; en dos (1,24\%) aparece acompañado de los términos tiempo libre y recreación.

\subsection{Recreación:}

Palabras derivadas como recreo, recreacional, etc., aparece como único término en los títulos de 32 trabajos (19,75\%).

Aparece junto a otro(s) término(s) en los títulos de nueve trabajos (6\%), asociado con: a) Ocio y tiempo libre: dos (1,24\%); b) Educación Física: 11 (6,79\%); c) Educación Física y Deporte: dos (1,24\%); d) Deporte, en uno (0,61\%); e) Comunitaria en uno $(0,61 \%)$; f) Actividad física: uno $(0,61 \%) ;$ g) Textos recreativos: uno (0,61\%); h) Tiempo libre: dos (1,24\%).

\subsection{Tiempo libre:}

No aparece como único término en ninguno de los títulos.

Aparece junto a otro(s) término(s) en los títulos de dos trabajos (1,24\%), ilustrados como 'ocio, tiempo libre y recreación'. Y utilizado de forma instrumental '...buen uso del tiempo libre' en dos (1,24\%).

Observaciones:

- El ocio y el tiempo libre no se presentan en ningún título como único término.

- Solo dos trabajos $(1,24 \%)$ incluyen en sus títulos como aspectos resaltantes a: 'ocio, tiempo libre y recreación'. En los restantes 52 (32,1\%) aparece el término recreación distribuido de la siguiente manera: en 32 (19,75\%) como término único y en 21 (12,96\%) asociado con otros términos.

\section{Autores(as)}

Tenemos que: a) todos los autores(as) son venezolanos; b) 33 autores(as) del total, están activos en investigación, y la nacionalidad de todos ellos es venezolana; c) Resulta llamativo la alta cantidad de autores(as) activos en investigación. 
Reyes, A., Altuve, E., \& Arandia, G. (2021). Investigación en recreación, ocio y tiempo libre en Venezuela. Enfoques subyacentes. Revista Ciencias de la Actividad Física UCM, 22(2), julio-diciembre, 1-21. http://doi.org/10.29035/rcaf.22.2.8

\section{Tercer Período: 2008-2010}

En el período comprendido entre los años 2008 y 2010, se presentan 31 trabajos (19,14\% del total), de los cuales:

\section{Tipo de material e institución que auspicia la investigación:}

Tabla 8

Caracterización de la producción de conocimiento en el período 2008-2010.

\begin{tabular}{|c|c|c|c|}
\hline Tipo de material & Institución & FR & $\%$ \\
\hline $\begin{array}{l}\text { Trabajo de Grado (Maestría en Enseñanza de la Educación } \\
\text { Física) }\end{array}$ & UPEL-IPC & 11 & 6,79 \\
\hline $\begin{array}{l}\text { Trabajo de Grado (Maestría en Enseñanza de la Educación } \\
\text { Física) }\end{array}$ & UPEL-Maracay & 5 & 3,01 \\
\hline Conferencias (Carlos Vera Guardia) & Sin filiación & 5 & 3,01 \\
\hline Libros (Universidad Federal de Mina Gerais) & Sin filiación & 2 & 1,24 \\
\hline $\begin{array}{l}\text { Trabajo de Grado (Maestría en Enseñanza de la Educación } \\
\text { Física) }\end{array}$ & UPEL-IPMALA & 1 & 0,61 \\
\hline Trabajo Anónimo & UNEFM & 1 & 0,61 \\
\hline Articulación (Publicación política) & $\begin{array}{l}\text { Red Socialista } \\
\text { de Venezuela }\end{array}$ & 1 & 0,61 \\
\hline Artículo (Revista "Práticas de Animação") & Sin filiación & 1 & 0,61 \\
\hline $\begin{array}{l}\text { Ponencia (IX Congreso Argentino y IV Latinoamericano de } \\
\text { Educación Física y Ciencias, de la Universidad de La Plata. } \\
\text { Argentina) }\end{array}$ & LUZ & 1 & 0,61 \\
\hline Libro (Editorial Apicuri) & LUZ & 1 & 0,61 \\
\hline Artículo (Revista Acción Motriz) & LUZ & 1 & 0,61 \\
\hline $\begin{array}{l}\text { Panel (III Foro Mercosur latinoamericano por la } \\
\text { Democratización del Deporte, la Educación Física y la } \\
\text { Recreación, Ecuador) }\end{array}$ & LUZ & 1 & 0,61 \\
\hline TOTALES & & 77 & 19,14 \\
\hline
\end{tabular}

Fuente: Elaboración propia.

\section{Observaciones:}

a) 16 trabajos $(9,87 \%)$ son tesis de grado de universidades venezolanas, de los cuales 15 (9,25\%) son de la misma universidad -UPEL-, específicamente de las sedes de la UPEL-Maracay, la UPEL-IPMALA y principalmente del núcleo UPEL-IPC, que aporta 11 (6,79\%); b) dos (1,24\%) son publicaciones de una revista electrónica de un centro de investigación de la UPEL-Maracay; c) uno $(0,61 \%)$ es un artículo de una publicación política nacional; d) cuatro (2,50\%) son publicaciones internacionales, de las cuales dos $(1,31 \%)$ son auspiciadas por una universidad y dos (1,31\%) por una organización científico-académica independiente. Fueron elaboradas por una profesora de UPEL-Maracay y por un profesor de LUZ; e) uno (0,61\%) es un libro publicado por la Editorial Apicuri. Rio de Janeiro-Brasil: Consejo Nacional de Pesquisa de Brasil (CNPQ) elaborado por un profesor de la Universidad del Zulia; f) uno $(0,61 \%)$ es una ponencia en el IX Congreso Argentino y IV Latinoamericano de Educación Física y Ciencias, de la Facultad de Humanidades 
Reyes, A., Altuve, E., \& Arandia, G. (2021). Investigación en recreación, ocio y tiempo libre en Venezuela. Enfoques subyacentes. Revista Ciencias de la Actividad Física UCM, 22(2), julio-diciembre, 1-21. http://doi.org/10.29035/rcaf.22.2.8

y Ciencias de la Educación de la Universidad de La Plata (Argentina), por un profesor de la Universidad del Zulia; g) cinco (3,01\%) son ponencias en conferencias, de las cuales dos son nacionales y tres son internacionales, dictadas por el profesor Carlos Veraguardia.

\section{Término resaltante y/o destacado en el título}

2.1. En 14 (8,64\%) se encuentran términos relevantes que aluden a lo operativo-instrumental: a) ocho (4,94\%) tienen palabras como: 'Programa', 'proyecto', 'planes, actividades'; b) tres tienen palabras como: (1,85\%) 'Estrategias', 'manejo'; c) tres (1,85\%) tienen palabras como: 'Uso constructivo', 'construcción del perfil', 'gestión del uso'.

2.2. En 17 (10,49\%\%) aparecen términos relevantes que aluden a un criterio analítico mucho más amplio: a) En uno (0,61\%): 'Modelos de análisis...'; b) En uno (0,61\%): 'Ocio, recreación, Estado y Revolución'; c) En uno (0,61\%): 'La recreación en Venezuela...'; d) En uno (0,61\%): 'Libro de texto para la asignatura de fundamentos teóricos'; e) En uno (0,61\%): 'Propuesta de maestría en recreación...; f) En uno (0,61\%): 'La recreación y el desarrollo endógeno...'; g) En uno (0,61\%): 'Una visión...; h) En uno (0,61\%): 'Ocio, tiempo libre y recreación...; I) En uno (0,61\%): 'equipamientos culturales'; j) En uno (0,61\%): '...construcción colectiva de un Sistema Nacional Bolivariano de Juego, Educación Física, Deporte y Recreación...; k) En uno (0,61\%) 'Aproximación a una metodología de análisis...'; L) En dos (1,24\%): 'infraestructura deportiva y recreativa...'; m) En uno (0,61\%): 'Deporte, recreación y desarrollo humano'; n) En uno (0,61\%): 'La educación física y el alto rendimiento en la época actual...; o) En uno (0,61\%): 'Nuevas tendencias en la gestión del deporte y la recreación'; p) En uno (0,61\%): 'Tendencias en la gestión de las instalaciones para deporte y recreación'.

Observaciones:

- Los títulos de 14 trabajos (8,64\%), resumen o reducen la investigación a lo operativo, instrumental, ejecución de acciones, referidos a asuntos muy puntuales: programas, proyectos, planes actividades, estrategias, etc.

- Asomándose con criterios analíticos un poco más amplios, se encuentran los títulos de 11 $(7,18 \%)$

- De los $17(10,49 \%)$ con criterios analíticos un poco más amplios en sus títulos: ocho (4,93\%) son publicaciones internacionales, auspiciadas por una universidad y por organizaciones científico-académica independientes; dos (1,24\%) son publicaciones de una revista electrónica de la UPELMaracay; uno (0,61\%) es un trabajo de la UPELMaracay; uno (0,61\%) es una tesis de grado de UPEL-IPC; y uno (0,61\%) es un artículo en una publicación política.

\section{Presencia de los términos recreación, ocio y tiempo libre en los títulos}

\subsection{Ocio}

No aparece como único término en ninguno de los títulos.

Aparece junto a otro(s) término(s) en los títulos de cuatro trabajos (2,47\%), asociado con: a) Recreación en uno (0,61\%); b) Tiempo libre, en uno (0,61\%); c) Tiempo libre y recreación, en dos (1,24\%).

\subsection{Recreación}

Palabras derivadas como recreo, recreacional, etc., aparece como único término en los títulos de 13 trabajos (7,41\%).

Aparece junto a otro(s) término(s) en los títulos de 14 trabajos (8,64\%), asociada con: a) Educación Física y deporte, en cuatro (2,47\%); b) Ocio, en uno (0,61\%); c) Actividad física y tiempo libre, en uno (0,61\%); d) Ocio y tiempo libre, en dos (1,24\%); e) Tiempo libre, en uno $(0,61 \%) ;$ f) Deporte, en cinco $(3,1 \%)$.

No aparece en el título de dos trabajos (1,24\%). 


\subsection{Tiempo libre:}

Aparece como único término en uno $(0,61 \%)$.

Aparece junto a otro(s) término(s) en los títulos de cinco trabajos (3,09\%), asociado con: a) Recreación, en dos (1,24\%); b) Ocio, en uno $(0,61 \%) ;$ c) Ocio y recreación, en dos (1,24\%)

Observaciones:

- La recreación se mantiene como centro de la investigación al estar presente exclusivamente en los títulos de 13 trabajos (7,47\%), asociada a otro término en 14 (8,64\%) y ausente en sólo dos (1,24\%).

- El tiempo libre complementa el objeto de estudio -en términos operativos e instrumentales- estando presente en seis (3,70\%) títulos. Mientras el ocio, se asoma tímidamente, asociado a otros términos en cuatro $(2,47 \%)$ títulos

\section{Autores(as)}

Para este período un total de 23 investigadores Todos los autores son venezolanos. La cantidad de investigadores activos en el período, con un total de 17.

\section{DISCUSIÓN}

En Venezuela, la investigación sobre recreación, ocio y tiempo libre, se ha realizado generalmente en las universidades, teniendo como punto de partida o referencia las Escuelas, Facultades y/o Departamentos de Educación Física y/o Deporte existentes, sumándose en los últimos períodos los programas de formación avanzada y algunas unidades de investigación (de las mismas universidades), tal y como se confirma en Reyes (2020).

En el primer período (1991-1999), con la rectoría de la Maestría en Recreación de LUZ y la Maestría en Educación Física de la UPEL-IPC, la corriente predominante es la positivista, cuyo enfoque es el empírico-analítico (en la propuesta de Habermas, 1988). Asociado el paradigma positivista con la lógica eurocéntrica (Christiansen, 2017, Gibert, 2017), ha venido centrando su foco en el contexto de la recreación, manejando e imprimiendo una especie de laxitud e imprecisión conceptual. En esta tendencia, el objeto de estudio ha sido lo exclusivamente operativo, la ejecución de acciones, el 'cómo hacer', la cantidad de actividades, la cantidad de participantes; el ocio no es motivo de análisis, el tiempo libre se percibe instrumentalmente e implícitamente es condenado y hay muy poca precisión en el uso del concepto 'recreación'. Esto es compatible con los resultados de una investigación realizada por Tabares et al. (2014), en la que se advierte que el paradigma positivista es el que ha prelado en América Latina y también en Venezuela con respecto al campo de estudios de la recreación, el ocio y el tiempo libre. De igual manera, coincide con Reyes (2020), en el que se destaca la preeminencia de este tipo de abordajes en las primeras etapas de la investigación en este campo a partir del desarrollo de los primeros programas de posgrado en el país. Además de ello, en un trabajo de caracterización de la producción científica en recreación en el Instituto Pedagógico de Maturín, (Reyes et al., 2019), también se advierte como hallazgo, la imprecisión en el abordaje conceptual de la recreación. Y Altuve (2009), en un estudio realizado en el período de estudio, ya había destacado que:

En Venezuela es extremadamente limitada la reflexión profunda, seria, vibrante, sobre el problema del ocio, el tiempo libre, la recreación, es casi inexistente la reflexión y el debate sobre estos temas, la universidad lo hace poco y el Estado casi nada. Es necesario aceptar y asumir que, en Venezuela, históricamente, no se han presentado los grandes debates teóricoconceptuales sobre el ocio, el tiempo libre y la recreación, que se han adelantado en otros países de América Latina (p. 381).

Todo ello sirve como insumo para considerar que, en Venezuela, como en buena parte de América Latina, este sigue siendo un problema que dificulta el consenso y genera obstáculos no 
menores en los procesos de consolidación de la recreación como campo (Quintero, 2021). A lo que sostiene Artazcoz (2003):

(...) la recreación como campo de estudio, es un territorio a ser explorado que necesita de un amplio marco conceptual para su desarrollo. En efecto, debido a la pluralidad de significados que se le otorgan y a sus diversas manifestaciones, requiere de construcciones teóricas (sec. 1/1).

En el segundo período (2000-2007), se mantiene el positivismo como corriente predominante (enfoque empírico-analítico) en la investigación liderada por los trabajos de grado de la Maestría en Enseñanza de la Educación Física de la UPEL-IPC, existiendo adicionalmente sólo dos trabajos presentados como ponencias en congresos internacionales desde la USB, institución que no presenta carrera asociada a la recreación, y tampoco a la carrera de educación física, deporte y recreación, ni a nivel de Licenciatura, tal y como es la tendencia en Venezuela y América Latina. Sigue centrada en la recreación y, principalmente, se reduce lo operativo, a la ejecución de acciones: programas, proyectos, actividades, diseño, elaboración de planes y medición.

Y en el tercer período (2008-2010) se diversifica el origen de los trabajos procediendo de la UPEL (IPMALA; IPC; IPMAR), Universidad Nacional Experimental Francisco de Miranda de Coro-Estado Falcón y Universidad del Zulia, presentándose algunos en publicaciones internacionales y en la Revista Electrónica Actividad Física y Ciencias del Centro de Investigación en Educación Física, Salud, Deporte, Recreación y Danza (EDUFISADRED), de la UPEL-IPC Maracay. Aun cuando se mantiene la preeminencia y hegemonía de la corriente positivista, se asiste a la gestación de una visión con criterios analíticos un poco más amplios, pretendiendo exceder el marco operativoinstrumental, empezando a perfilarse lo teóricoepistemológico y metodológico como preocupación. Así, hay trabajos que se abordan desde los enfoques, histórico-hermenéutico y crítico-social. Esto se consolida al comparar con los resultados de estudios realizados por Sánchez et al. (2018), quienes sostienen que los enfoques metodológicos cualitativos y con enfoques hermenéutico-críticos, han venido en auge en los últimos tiempos en ciencias sociales.

La recreación se mantiene como centro de la investigación, el tiempo libre complementa el objeto de estudio y la consideración sobre el ocio se asoma tímidamente. Estos planteamientos con coherentes con los resultados de trabajos presentados por Guerrero (2009), Molina et al. (2019), Reyes (2019, 2020) y Tabares et al. (2014), en el sentido de que, si bien es cierto, sigue siendo predominante la investigación con enfoque positivista, comienzan a aparecer trabajos desde otras perspectivas metodológicas con la diversificación de nuevos programas y la incorporación de nuevos investigadores. Al mismo tiempo, Reyes (2020) destaca que, el relacionamiento con investigadores de otros países, la generación de redes y la incorporación en grupos de investigación de carácter regional, ha servido para que la investigación en el campo de estudios de la recreación, el ocio y el tiempo libre, tribute a incluir e incorporar otros abordajes de investigación y nuevas metodologías. Justo con respecto a este último tema, es posible advertir semejanzas con lo que ya destacan Peralta et al. (2015), en lo concerniente a la necesidad de incorporar nuevas alternativas metodológicas en el campo de la recreación, el ocio, lazer y tiempo libre. Ello, habida cuenta que el crecimiento del campo implica nuevos abordajes metodológicos que permitan otras comprensiones y otras interpretaciones de estos fenómenos socioculturales. Abordajes que a la vez ofrezcan posibilidades otras a las generadas desde la lógica del positivismo. Además, estas perspectivas de investigación coinciden con los primeros esbozos del Plan Nacional de Recreación para el Buen Vivir que luego pasa a denominarse Plan Nacional de Recreación para el Vivir Bien, el surgimiento del Movimiento Nacional de Recreadores, en asociación con esfuerzos de parte 
del equipo de asesores del plan, que generan un eje de investigación en el campo de la recreación y para lo cual convocan a universidades, unidades y líneas de investigación (Reyes, 2020).

Esto permite considerar entonces que existen dos grandes etapas de la investigación en recreación, ocio y tiempo libre en Venezuela:

7. Positivista-instrumental-operativa: priorizando un enfoque empírico analítico, se inaugura en Venezuela con la instauración de los estudios técnicos en el campo de las ciencias sociales, con profesores recién llegados de maestrías y especializaciones de perfil técnico y gerencial, especialmente de los Estados Unidos (Reyes, 2018). Privilegia la racionalidad instrumental, el tecnicismo, el vaciado político de los elementos socioculturales, la simpleza investigativa (en tanto la mayoría de los trabajos presentados se basan en encuestas de intereses lúdicos de grupos específicos, sin que la construcción y la aplicación de los programas se corresponda con las verdaderas necesidades e intereses, sino que se ajusta a las expectativas previas y los correlatos preestablecidos de los investigadores). Por ejemplo, el término 'reflexión crítica' (al más puro estilo de Habermas, 1988) es ignorado en los resúmenes de los trabajos de investigación en tanto lo que orienta las investigaciones se aproxima al desarrollo de planes recreativos, programas esporádicos (o relámpagos), manuales de instrucciones, cancioneros, programas específicos, etc. Temas asociados a políticas públicas no son considerados en el período en estudio, o evaluación de políticas públicas, enfoques sociocríticos, estudios de carácter cualitativo, o incluso partiendo de ejercicios interdisciplinares con otros campos más allá de la educación como la sociología, la antropología, política, religión, etc. Siendo de esta forma, la tendencia que existía en el país en el orden de la investigación en las ciencias fácticas inunda las previsiones investigativas que iban naciendo aún en el campo de las ciencias sociales (Reyes, 2018).

En Venezuela ha sucedido que los programas de formación avanzada (aquellos que consideraron la recreación dentro de sus líneas de interés), comenzaron sin existir un acervo de investigación en esos campos en el país. Entonces, se crearon los programas, se aprobó la creación de líneas de investigación sin tener sustento ni insumos que permitieran sostener la producción académica, y se impulsó el desarrollo de investigación muy básica como requisito de titulación. Así, la investigación en el campo de la recreación se genera tras la instauración de programas de formación avanzada (postgrados varios) por la necesidad de formar personal con perfiles técnicos y gerenciales en el campo. Incluso, primero surgen los programas de formación avanzada, y después, por pedido de las autoridades ministeriales en materia de educación universitaria (anteriormente conocida en Venezuela como educación superior), surgen las unidades y las líneas de investigación. Por supuesto, lo que debía ocurrir desde el principio es que la investigación diera paso a líneas de investigación, y estas líneas de investigación dieran paso a la formación de programas. Pero no fue eso lo que ocurrió (Reyes, 2018).

Esa tendencia positivista, instrumental y operativa se pone en escena en los programas de formación, las asignaturas y los perfiles de los egresados. Esto es, privilegiándose el hacer, la planificación, la ejecución de programas, planes y proyectos, sin la interpelación crítica de los haceres, las prácticas, los discursos, los sentidos y los significados, los contextos y las formas de investigar. Además, se ponía en escena desde la generación de los mismos títulos de investigación, los autores que son consultados de manera genérica, las formas de hacer la investigación, la imposición de un método ('el' método) a manera de recetario (una sola mirada de hacer investigación), la endogamia en la misma construcción de los consejos y las comisiones para evaluación de trabajos, entre otros.

2. Crítica-analítica-totalizadora: posicionando los enfoques, histórico-hermenéutico y crítico-social, surge a mediados de la primera década del siglo XXI y se mantiene como minoritaria. Excediendo el marco operativo-instrumental, maneja criterios 
analíticos mucho más amplios, comprendiendo hasta lo teórico-epistemológico y metodológico expresado de manera manifiesta como preocupación central. Incorpora, además de la recreación, el ocio y el tiempo libre como objetos de estudio. Comienzan a preocupar temas como el estudio de la recreación y el ocio como campo, las políticas públicas y metodologías de análisis de políticas públicas, episteme en el campo de la recreación, el ocio y el tiempo libre, estudios comparados, abordajes metodológicos mixtos, sistematización de experiencias, entre otros elementos.

\section{CONCLUSIONES}

Considerando que el estudio implica un rango de tiempo establecido entre 1999 y 2010, tendríamos que la investigación formal en recreación, ocio y tiempo libre inicia en Venezuela prácticamente con la instauración de programas de formación avanzada en recreación en el país, iniciando la década de los años 90' en el siglo XX, esto, sin desconocer algunas iniciativas de investigadores particulares. Básicamente, la investigación en estos campos deviene de universidades y centros de investigación que se van creando con el paso del tiempo, pero genéricamente se hace en el contexto universitario.

La investigación en recreación, ocio y tiempo libre en el período en estudio en Venezuela viene determinada por perfiles de formación en los programas de formación avanzada que se encuentran orientados al campo de la programación de actividades, el campo gerencial y a la medición de indicadores. Esto se suma a la orientación metodológica de los trabajos de grado (generalmente) y como extensión a los artículos publicados en revistas, a las ponencias $y$ conferencias publicadas en memorias de eventos, que tienen una tendencia positivista e instrumental (enfoque empírico-analítico).
La tendencia positivista e instrumental es la que ha imperado en la investigación en los campos de la recreación, el ocio y el tiempo libre en Venezuela, sin embargo, a partir del tercer período en estudio, ha comenzado a visualizarse la irrupción de otros abordajes metodológicos, en especial de una perspectiva sociocrítica, coincidiendo con procesos importantes en Venezuela como el surgimiento del primer Plan Nacional de Recreación, el surgimiento del Movimiento Nacional de Recreadores, la creación de nuevos programas de formación avanzada en el país desde los que se asumen otras metodologías de investigación, entre otros procesos.

Los enfoques, histórico-hermenéutico y críticosocial, inauguran una etapa que, va a consolidarse con el correr del tiempo, y que se posiciona como una alternativa en el marco de la investigación en recreación, ocio y tiempo libre en Venezuela y América Latina.

\section{Prospectiva}

Se tiene como prospectiva de este estudio culminar una segunda etapa de la indagación que permita considerar unidades de análisis entre 2011 y 2020, a fin de tener una panorámica mucho mayor con respecto a la investigación en recreación, ocio y tiempo libre en Venezuela durante el siglo XXI.

\section{Conflicto de intereses}

Los autores declaran no tener conflicto de intereses.

\section{Agradecimientos}

Los autores agradecen por la ayuda y colaboración a las siguientes personas e instituciones: Gladys Guerrero, Jackova Castillo, Ramón Enrique Reyes, de la UPEL; a la Biblioteca de Postgrado de la Facultad de Humanidades y Educación de LUZ; a Bárbara Ufre, de LUZ; a Gerardo Jiménez e Isabel Altuve. 


\section{REFERENCIAS BIBLIOGRÁFICAS}

Altuve, E (2009). Ocio, recreación, Estado y revolución. En C. Gomes, (Coord.), Lazer na América latina/Ocio y Recreación en Latinoamérica, (pp. 214-230). UFMG.

Arias M., M., \& Navarro C., M. (2017). Epistemología, Ciencia y Educación Científica: premisas, cuestionamientos y reflexiones para pensar la cultura científica. Revista Electrónica Actualidades Investigativas en Educación, 77(3),

http://dx.doi.org/10.15517/aie.v17i3.29878

Artazcoz, M. (2003). Una mirada desde los albores de la filosofía occidental para pensar las dimensiones constitutivas de la recreación. [Ponencia] III Simposio Nacional de Vivencias y Gestión en Recreación. Bogotá, Colombia.

http://www.redcreacion.org/documentos/si mposio3vg/MMArtazcoz.html

Benítez G., J., Cadenas S., M., Campón C., O., Espartero V., A., \& Muñoz R., E. (2015). Evolución del concepto de recreación y sus beneficios en diferentes poblaciones. Revista Heducasport, (0), 49-62. https://revistaheduca.files.wordpress.com/2 015/03/3_-articulo-quino-benitez.pdf

Berkin, S. C. (2019). Producción horizontal del conocimiento. CALAS.

Bourdieu, P. (1999). Razones prácticas sobre la teoría de la acción. (2ª ed). Anagrama.

Broncano, F. (2020). Teoría y práctica de las fraternidades epistémicas. Dilemata, 12(33), $11-21$.

https://www.dilemata.net/revista/index.ph p/dilemata/article/view/412000343

Carreño C., J. M., Díaz V., A., López D., S., \& Reyes, J. A. M. (2019). ¿Qué se investiga en formación docente en educación física y en recreación?. Retos, 36, 3-8. https://recyt.fecyt.es/index.php/retos/article /view/51456/42090
Carreño-Cardozo, J. M., Rodríguez-Cortés, A. B., \& Gutiérrez-Africano, P. (2014). Lineamientos de intervención de la recreación en la escuela. Magis, Revista Internacional de Investigación en Educación, 6(13), 83-97. https://doi.org/10.11144/Javeriana.M613.LIRE

Castro-Gómez, S. (2000). Ciencias sociales, violencia epistémica y el problema de la invención del otro. CLACSO.

Cifuentes G., R. M. (2011). Diseño de proyectos de investigación cualitativa. NOVEDUC.

Coello V., E., Balbeíto, N. B., \& Reyes O., Y. (2012). Los paradigmas cuantitativos y cualitativos en el conocimiento de las ciencias médicas con enfoque filosófico-epistemológico. Edumecentro, $\quad 4(2), \quad$ 137-146. http://scielo.sld.cu/pdf/edu/v4n2/edu17212.p df

Conde, A., Debellis, M., \& Moreira, L. (2016). Un área temática de la educación no formal: recreación, ocio y tiempo libre. Comisión Nacional de Educación no Formal del Ministerio de Educación y Cultura. https://www.conenfor.edu.uy/innovaportal/ file/5025/1/recreacion_ocio_y_tiempo_libre. $\mathrm{pdf}$

Christiansen, M. L. (2017). Epistemología Depredadora y Paz Violenta. Consideraciones Filosóficas sobre la Construcción de una Justicia Cognitiva. International and Multidisciplinary Journal of Social Sciences, 6(3), 281-306. https://doi.org/10.17583/rimcis.2017.2992

Duque B., H. C., Franco B., S. A., \& Escobar C., A. (2008). Fundamentos conceptuales del ocio crítico desde una perspectiva latinoamericana. [Tesis de Maestría no publicada]. Universidad de Antioquia, Colombia. 
Elizalde, R., \& Gomes, C. (2010). Ocio y recreación en América Latina: conceptos, abordajes y posibilidades de resignificación. Polis, Revista Latinoamericana, 26, 1-19. https://journals.openedition.org/polis/64

Gibert, J. (2017). La Redefinición de las Identidades de los Cientistas Sociales Latinoamericanos: ¿Hacia un Nuevo Colonialismo Intelectual? Revista Latinoamericana de Educación Inclusiva, 37(7), 35-55 https://scielo.conicyt.cl/pdf/rlei/v11n1/art04.p df

Giuliano, F. (Comp.). (2018). ¿Podemos pensar los no europeos? Ética decolonial y geopolíticas del conocer. Ediciones del Signo.

Gomes, C. L. (2014). El ocio y la recreación en las sociedades latinoamericanas actuales. Polis, Revista Latinoamericana, 13(37), 363$384 . \quad$ http://dx.doi.org/10.4067/s071865682014000100020

Guerrero, G. (2009). La recreación en Venezuela, un valor para el equilibrio social. En C. Gomes, E. Osorio, L. Pinto, R. Elizalde, \& A. M. Oliveira (Eds.). Lazer na América Latina / Ocio y Recreación en Latinoamérica. (pp. 194204). UFMG

Habermas, J. (1988). La lógica de las ciencias sociales. Tecnos.

Jandrić, P., \& Hayes, S. (2019). The postdigital challenge of redefining academic publishing from the margins. Learning, Media and Technology, 44(3), 381-393. https://doi.org/10.1080/17439884.2019.15858 74

Lander, E. (Comp.). (2000). La colonialidad del saber: eurocentrismo y ciencias sociales. Perspectivas latinoamericanas. CLACSO.

Mangez, C., \& Mangez, E. (2011). Producing Dangerous Knowledge: researching knowledge production in Belgium. European Educational Research Journal, 10(2),
Molina B., V. A., Hurtado C., A., Tabares F., J. F., \& Franco B., S. A. (2019). Metodologías otras para el estudio del ocio y la recreación en América Latina. Puertabierta Editores.

Ortiz O., A. (2015). Enfoques y métodos de investigación en las ciencias sociales y humanas. Ediciones de la U. https://edicionesdelau.com/producto/enfo ques-y-metodos-de-investigacion-en-lasciencias-sociales-y-humanas2/\#iLightbox[]/0

Osorio, E. (2014). El campo de la recreación y el ocio: su configuración como un movimiento por la emancipación y el buen vivir. En Universidad de Colima (Ed.), Memoria en extenso del Encuentro Internacional de Organismos Expertos en Cultura Física, (pp. 30-41). Universidad de Colima.

Osorio, E. (2019). Asuntos presentes del campo de la recreación y el ocio. Casa de las preguntas. https://www.academia.edu/42682181/ASUN TOS_PRESENTES_DEL_CAMPO_DE_LA_RE CREACI\%C3\%93N_ASUNTOS_PRESENTES_ DEL_CAMPO_DE_LA_RECREACI\%C3\%93N _ESTUDIOS_LATINOAMERICANOS_DE_OC 10

Osorio, F. (2007). Desde dónde se escriben las ciencias sociales al comienzo del siglo XXI. En F. Osorio (Ed.), Epistemologías de las ciencias sociales. Breve manual, (pp. 7-12). LOM. 
Peralta A., R.; Medina V., R. T.; Osorio C., E., \& Salazar C., C. M. (2015). Aproximaciones para la construcción del campo de la recreación en América Latina. Puertabierta Editores. https://www.academia.edu/15647627/Aprox imaciones_para_la_construcci\%C3\%B3n_d el_campo_de_la_recreaci\%C3\%B3n_en_Lat inoam\%C3\%A9rica

Quintero R., M. (2014). La recreación: un acto social para la construcción de lo humano. Revista Ímpetus, 8(1), 35-41. http://dx.doi.org/10.22579/20114680.27

Quintero R., M. (2021). Aporte a la construcción del concepto de recreación como campo de conocimiento desde las realidades latinoamericanas. Revista Con-Ciencias del Deporte, 3(1), 106-130. http://revistas.unellez.edu.ve/index.php/rcc d/article/view/1276

Ramos, C. A. (2015). Los paradigmas de la investigación científica. Avances en Psicología, 23(1), 9-17. https://doi.org/10.33539/avpsicol.2015.v23n1.1 67

Reyes, A. (2011). Plan Nacional de Recreación para el Vivir Bien. https://www.aporrea.org/actualidad/a13529 3.html

Reyes, A. (2014). Consideraciones básicas sobre la asunción de conocimiento. Praxis \& Saber, 5(9), 103-126. https://doi.org/10.19053/22160159.2702

Reyes, A. (2015). Políticas públicas en el marco de una nueva cultura de la recreación. Humanartes, $\quad$ 4(7), 8-32. https://www.researchgate.net/publication/ 329572301_POR_UNA_NUEVA_CULTURA_ DE_LA_RECREACION
Reyes, A. (2018). Pensar la recreación. Entre tensiones y paradojas socioculturales de la América Latina. Una apología en claves heréticas. [Sesión de conferencia]. $8^{\circ}$ Conferencia Latinoamericana y Caribeña de Ciencias Sociales. Primer Foro Mundial del Pensamiento Crítico. Buenos Aires, Argentina.

https://www.clacso.org.ar/conferencia2018/ presentacion_ponencia.php?ponencia=201 712308116-8920-pi

Reyes, A. (2020). Recreación en Venezuela. Insumos para el debate. Red Venezolana de Investigación e Innovación en Recreación; Instituto Municipal de Recreación de Cabimas; Universidad Adventista de Chile. https://www.unach.cl/wp-

content/uploads/2020/08/Recreaci\%C3\%B 3n-en-Venezuela.-Insumos-para-el-

debate.pdf

Reyes, A., Zapata, E., \& Altuve, E. (2019). Caracterización de la producción de conocimiento en Recreación en el Instituto Pedagógico de Maturín "Antonio Lira Alcalá". Revista Actividad Física y Ciencias, $77(2)$, 129-146. https://revistas.upel.edu.ve/index.php/activi dadfisicayciencias/article/view/8888

Ruiz R., D. L. (2018). La noción de dispositivo y comunidad epistémica en la explicación de la formación de investigadores en el campo educativo, hacia un ejercicio epistemológico. Sincronía, (73), 102-114, http://sincronia.cucsh.udg.mx/pdf/73/a5_10 2_114.pdf

Sánchez-Gómez, M. C., Martín-Cilleros, M. V., Costa, A. P., \& García P., F. J. (2018). Posicionamiento de la investigación en ciencias sociales. RISTI - Revista Ibérica de Sistemas e Tecnologias de Informação, (28), 102-113. http://dx.doi.org/10.17013/risti.28.102-113

Fernández, J. F. T., Bedoya, V. A. M., Gerlero, J., Bestrad, P., Lazzarotti Filho, A., Dias, C., Morales, E. P., Conde, L., Martinez, E., Altuve, 
E., \& Reyes, A. (2014). Producción de conocimiento en ocio, recreación y tiempo libre en América Latina. LICERE - Revista Do Programa De Pós-graduação Interdisciplinar Em Estudos Do Lazer, 17(2), 192-221. https://doi.org/10.35699/19813171.2014 .854

Tabares, J. F. (2011). El conocimiento del ocio en las sociedades periféricas. Análisis de la producción científica sobre ocio, recreación y tiempo libre en Colombia [Tesis doctoral publicada], Universidad de Deusto, España. https://dialnet.unirioja.es/servlet/tesis?codi go $=118903$

Vasco, C. E. (1985). Tres Estilos de Trabajo en las Ciencias Sociales. Universidad Nacional de Colombia. https://aprendeenlinea.udea.edu.co/Ims/m oodle/pluginfile.php/175197/mod_resource/ content/O/Tres_estilos_de_trabajo_en_las_ Ciencias_Sociales.pdf
Velasco C., A. (2018). Condiciones epistémicas para la creación de conocimiento en ciencias humanas. Limite. Revista Interdisciplinaria de Filosofía y Psicología, 13(41), 46-59. http://dx.doi.org/10.4067/s071850652018000100046

Villoro, L. (1982). Creer, saber, conocer. Siglo XXI.

Waichman, P. (1998). Acerca de los enfoques en recreación. [Ponencia]. V Congreso Nacional de Recreación Coldeportes Caldas, Universidad de Caldas, Colombia. http://www.redcreacion.org/documentos/c ongreso5/PWaichman2.htm

Dirección para correspondencia

Alixon David Reyes Rodríguez

Doctor en Educación

Grupo de Estudios AFSYE de Pedagogía en Educación Física y Programa de Magíster en Ciencias de la Motricidad Humana de la Universidad Adventista de Chile

Chillán, Chile.

Dirección postal: Pasaje Villa el Bosque № 16.

Chillán, Chile

ORCID ID: https://orcid.org/0000-0001-9857-0930

Contacto:

alixdavid79@gmail.com

Recibido: 16-11-2020

Aceptado: 14-07-2021

Este obra está bajo una Licencia de Creative Commons

Reconocimiento-NoComercial-Compartirlgual 4.0 Internacional. 